\title{
Traditional Chinese Medicine in Cancer Care: An Overview of 5834 Randomized Controlled Trials Published in Chinese
}

Integrative Cancer Therapies

Volume 20: I-12

(C) The Author(s) 2021

Article reuse guidelines:

sagepub.com/journals-permissions DOI: $10.1177 / 15347354211031650$

journals.sagepub.com/home/ict

(SSAGE

\author{
Chun-Li Lu, PhD ${ }^{*}$ (D), Xun Li, PhD ${ }^{1 *}$, Hong-Mei Zhou, BA², Chi Zhang, BA', \\ Ya-Yue Yang, BA ${ }^{3}$, Ru-Li Feng, BA ${ }^{4}$, Chao-Jun Long, BA', Fang-Yuan Deng, BA', \\ Jing-Chun Li, BA ${ }^{2}$, Ze-Ming Cao, BA', Qi-Yuan Mao, BA ${ }^{5}$, Jin-Pu Zhu, BA', \\ Yan-Fei Hong, BA', Shou-Yu Huang, BA ${ }^{6}$, Jia-Ying Qiu, BA ${ }^{7}$, Yi-Xiu Liu, BA ${ }^{4}$, \\ Ying Wang, MMS ${ }^{8}$, Yu-Qian Yan, MMS', Jia-Min Dong, BA', Yu-Xin Luo, BA', \\ Yun-Meng Chen, BA ${ }^{4}$, Ying-Jie Guan, BA', Lisa Susan Wieland, MPH, PhD ${ }^{10}$, \\ Nicola Robinson, PhD' ', and Jian-Ping Liu, PhD, MD'
}

\begin{abstract}
Background: Traditional Chinese medicine (TCM) is widely integrated into cancer care in China. An overview in 20II identified 2384 randomized and non-randomized controlled trials (RCTs, non-RCTs) on TCM for cancer published in the Chinese literature. This article summarizes updated evidence of RCTs on TCM for cancer care. Methods: We searched 4 main Chinese databases: China National Knowledge Infrastructure, Chinese Scientific Journal Database, SinoMed, and Wanfang. RCTs on TCM used in cancer care were analyzed in this bibliometric study. Results: Of 5834 RCTs (477 I 57 cancer patients), only 62 RCTs were indexed in MEDLINE. The top 3 cancers treated were lung, stomach, and breast cancer. About 4752 RCTs (8I.45\%) tested TCM combined with conventional treatment, and 1082 RCTs (I8.55\%) used TCM alone for treating symptoms and side-effects. Herbal medicine was the most frequently used TCM modality (5087 RCTs; $87.20 \%$ ). The most frequently reported outcome was symptom improvement (37I2 RCTs; 63.63\%) followed by quality of life (2725 RCTs; 46.7I\%), and biomarkers (2384 RCTs; 40.86\%). The majority of RCTs (405I; 69.44\%) concluded there were beneficial effects using either TCM alone or TCM plus conventional treatment compared with conventional treatment. Conclusion: Substantial randomized trials demonstrated different types/stages of cancer were treated by various TCM modalities, alone or in combination with conventional medicine. Further evaluation on the effects and safety of TCM modalities focusing on outcomes such as quality of life is required.
\end{abstract}

\section{Keywords}

Traditional Chinese medicine, cancer, Chinese literature, randomized controlled trials, clinical evidence, bibliometric analysis

Submitted April 5, 2020; revised June II, 2021; accepted June 23, 202 I

\section{Background}

Cancer draws wide attention all over the world, due to its rapid rise in incidence and mortality, especially in low and middle-income countries. ${ }^{1}$ In 2018, global cancer statistics suggested that there were 18.1 million new cancer cases and 9.6 million cancer deaths worldwide ${ }^{2}$ and WHO has predicted that the number of new cases will increase to 21.6 million annually by $2030 .{ }^{3}$ There is an overwhelming need for patients to adopt and implement high-quality and resourceappropriate cancer treatment. ${ }^{4}$ Although medicine has made advances in cancer treatment (eg, surgery, chemotherapy, and radiation therapy) patient quality of life is affected by symptoms associated with both cancer and cancer treatment, and patients require quality post-treatment cancer care. ${ }^{5}$ Many cancer patients wish to explore complementary or integrative approaches to improve symptoms and quality of life. In 1998, the Office of Cancer Complementary and Alternative Medicine (OCCAM) was established within National Cancer Institute (NCI) in United States of America identified 2 main systems of medicine, Chinese medicine and Indian (Ayurveda) medicine. ${ }^{6}$ They stated that current 
scientific evidence is required on the effectiveness and safety of complementary health approaches and the highest quality evidence is available. ${ }^{7}$

Traditional Chinese medicine (TCM), with thousands of years of history, is still developing in contemporary China. ${ }^{8}$ Many TCM modalities such as acupuncture, qigong, tuina (Chinese massage) are recommended in both conventional and Chinese clinical practice guidelines for cancer management. ${ }^{9-11}$ Systematic reviews and clinical practice guidelines appear to summarize that some TCM modalities are effective in reducing side-effects from chemotherapy and radiation therapy, and improving clinical symptoms and quality of life. ${ }^{10-14}$ The benefits of using TCM alone or in combination with conventional treatment requires further study. When considering exploring the effectiveness and safety of treatments involving TCM, validated outcomes should also be carefully considered.

The randomized controlled trial (RCT) is the most appropriate study design for evaluating therapeutic effectiveness and safety. Given the large numbers of TCM RCTs published in Chinese, it is helpful to provide a comprehensive investigation of the distribution of cancers studies, TCMs used as interventions and research outcomes. This can be achieved by identifying and categorizing the existing RCTs, especially when there is very limited way to access studies published in Chinese outside of China. Our previous publication review including RCTs and non-RCTs of TCM for cancer has been cited in more than 120 international articles and drawn much attention from peers. ${ }^{15}$ There is a global awareness about TCM and therefore it is worth summarizing and updating the current best evidence for TCM and its use in cancer management. Given the knowledge gap in the West on Chinese literature on TCM for cancer care, the aim was to systematically describe the general scope of TCM applications in cancer RCTs based on Chinese literature.

\section{Methods}

An updated systematic search of the literature was conducted for the 5 years since the previous overview. ${ }^{15}$ All identified RCTs were then examined to summarize patterns in the research literature for TCM and cancer.

\section{Data Sources and Searches}

The updated searches were performed in 4 main Chinese databases including China National Knowledge Infrastructure (CNKI), Chinese Scientific Journal Database (VIP), SinoMed, and Wanfang Database, from November 2011 to 2017. The search strategy was the same one which was used for the 2011 overview (see Supplemental Table 1). Language restriction was Chinese because the 4 databases are primarily in the Chinese language. For quality reasons, we restricted eligibility to RCTs and excluded uncontrolled trials and controlled trials which did not use randomization. About 2383 RCTs were included and 581 non-randomized clinical studies excluded which had been identified in the previous overview. Four authors (CL Lu, HM Zhou, Z Chi, and YY Yang) conducted the search in 4 databases on June 182017.

\section{Study Selection}

Three authors (CL Lu, Y Wang, and YQ Yan) screened the title and abstracts of the retrieved records, and examined the full text of potentially relevant records to identify the eligible trials. Two authors (CL Lu and HM Zhou) screened and selected the RCTs from the previous overview. ${ }^{15}$

\section{Eligibility Criteria}

The 4 databases searches included journal papers, conference proceedings, and dissertations. All studies were published with full texts.

Type of studies. All randomized controlled studies reporting that they used randomization (RCTs) were included. All articles available in full text were included, even conference proceedings.

\footnotetext{
'Beijing University of Chinese Medicine, Beijing, China

${ }^{2}$ Dongfang Hospital of Beijing University of Chinese Medicine, Beijing, China

${ }^{3}$ Fudan University, Shanghai, China

${ }^{4}$ Dongzhimen Hospital of Beijing University of Chinese Medicine, Beijing, China

${ }^{5}$ Guang'anmen Hospital of China Academy of Chinese Medical Sciences, Beijing, China

${ }^{6}$ Wangjing Hospital of China Academy of Chinese Medical Sciences, Beijing, China

${ }^{7}$ Xiyuan Hospital of China Academy of Chinese Medical Sciences, Beijing, China

${ }^{8}$ China Press of Traditional Chinese Medicine, Beijing, China

'University Hospital Zürich, Zurich, Switzerland

${ }^{10}$ University of Maryland School of Medicine, Baltimore, USA

"Institute of Health and Social Care, London South Bank University, London, UK

*These authors are the co-first authors who contributed equally to this work.
} 
Type of participants. All types of participants with diagnoses of cancer were included. There was no restriction on cancer type, including malignant tumors, malignant hematological disease and patients with precancerous conditions (such as chronic atrophic gastritis, myelodysplastic syndrome, and other potential precancerous lesions). Patients were diagnosed by image-guided diagnosis methods or other tumor detection and diagnostic methods.

Type of intervention. TCM modalities used alone or in combination with conventional treatment for all types of cancerrelated patients were included. There was no restriction in comparators.

Type of outcomes. There was no restriction on type of outcome assessed. Given the volume and breadth of literature, bibliometric analysis was used to identify the outcomes in the included TCM RCTs for cancer care, to identify the key areas, and gaps in knowledge.

\section{Data Extraction}

Two authors (X Li and JP Liu) designed the original structured data extraction form and improved the original version for the inclusion of new information and to facilitate analysis. Data collection included citation information, publication type and funding, inclusion/exclusion criteria, diagnostic criteria, study methodology, details of participants (clinical related indices), details of interventions and controls, TCM intervention details, TCM syndrome differentiation, outcome measurements, and study conclusions.

For the RCTs (2011-2017) update, we ran data extraction, data checking, and data cleaning to verify the extraction process and checking. For previously included RCTs (inception-2011), 2 authors (CL Lu and HM Zhou) selected the RCTs and completed their data sheets directly. All RCTs were then combined for data analysis.

\section{Data Analysis}

Three authors (CL Lu, HM Zhou, and Z Chi) performed the data analysis using WPS Office Excel (11.1.0.10463-release. https://www.wps.cn/.), and calculated counts and percentages and frequencies. If 1 study contained various types of cancers, it was counted more than once in the study.

\section{Results}

\section{Description of Included Studies}

Our previous and updated searches retrieved a total of 153056 citations of which 7970 citations were selected for full text review. The 2136 citations were excluded as they were not eligible for inclusion or were unavailable as full text. A total of 5834 RCTs involving 477157 participants were included. The flow diagram of the literature search and study selection is given in Figure 1.

All RCTs were published in 1984 or later. The numbers of RCTs increased rapidly during 2012 to 2016 (Figure 2). The low number for 2017 is likely due to the search being conducted in mid-2017 as well as the time lag in recording citations in electronic databases, and we estimate that the total number of studies published in 2017 would have been at least 900. Among 5834 studies, 5108 studies $(88.79 \%$ ) were published in journals, 616 were dissertations, and 110 were conference proceedings with full text. Of the 5108 journal articles, 2651 were published in a TCM journal, 440 in an integrative Chinese and conventional medicine journal, 1873 in a General journal, 116 in the Zhonghua journal series (the high-quality core journals published in China), and the remaining 28 in other Chinese journals (such as the journal hosted by a university). Only 62 studies could be identified in MEDLINE. All core journal articles were published having undergone a process of "peer review" and all dissertations conducted by Master and $\mathrm{PhD}$ students were strictly blindreviewed by at least 2 experts assigned by the Ministry of Education of the People's Republic of China. Due to missing information, the review process of conference proceedings could not be found. Although this is defined as grey literature, it is known that all conferences proceedings need to be reviewed by at least 1 expert to guarantee the study quality.

Only 1144 studies reported having funding. A total of 173 studies were supported by a national grant or project, 662 had a provincial-level grant/project, and 309 had lower level funding such as university-level grants and projects.

Comparing the 5834 RCTs, 1694 studies (29.04\%) reported the randomization methods in detail (including random number tables, stratified randomization, computer randomization, tossing a coin, and drawing straws), 194 studies $(3.33 \%)$ reported the blinding of participants, doctors, outcome assessors and statistical staff in detail. Among the 1144 studies (19.61\%) reporting funding, 520 studies $(45.45 \%)$ reported the randomization methods in detail, and 59 studies $(5.16 \%)$ reported blinding in detail. This indicated that better reporting of funding may be associated with better overall reporting of methods.

\section{Participants and Diseases}

Among 5834 RCTs (477157 participants), 3656 studies $(62.67 \%)$ reported the diagnostic criteria, among these studies 3158 reported the standard diagnostic criteria of cancer stage, including pathological examination and cytology examination. The remaining 2178 studies (37.33\%) did not report any details about diagnostic criteria, but only reported that in-patients diagnosed by the clinical department of oncology were included. About 3270 studies (56.05\%) reported their inclusion criteria and the remaining 2564 studies $(43.95 \%)$ failed to report in detail their inclusion information, often only in 1 sentence. 


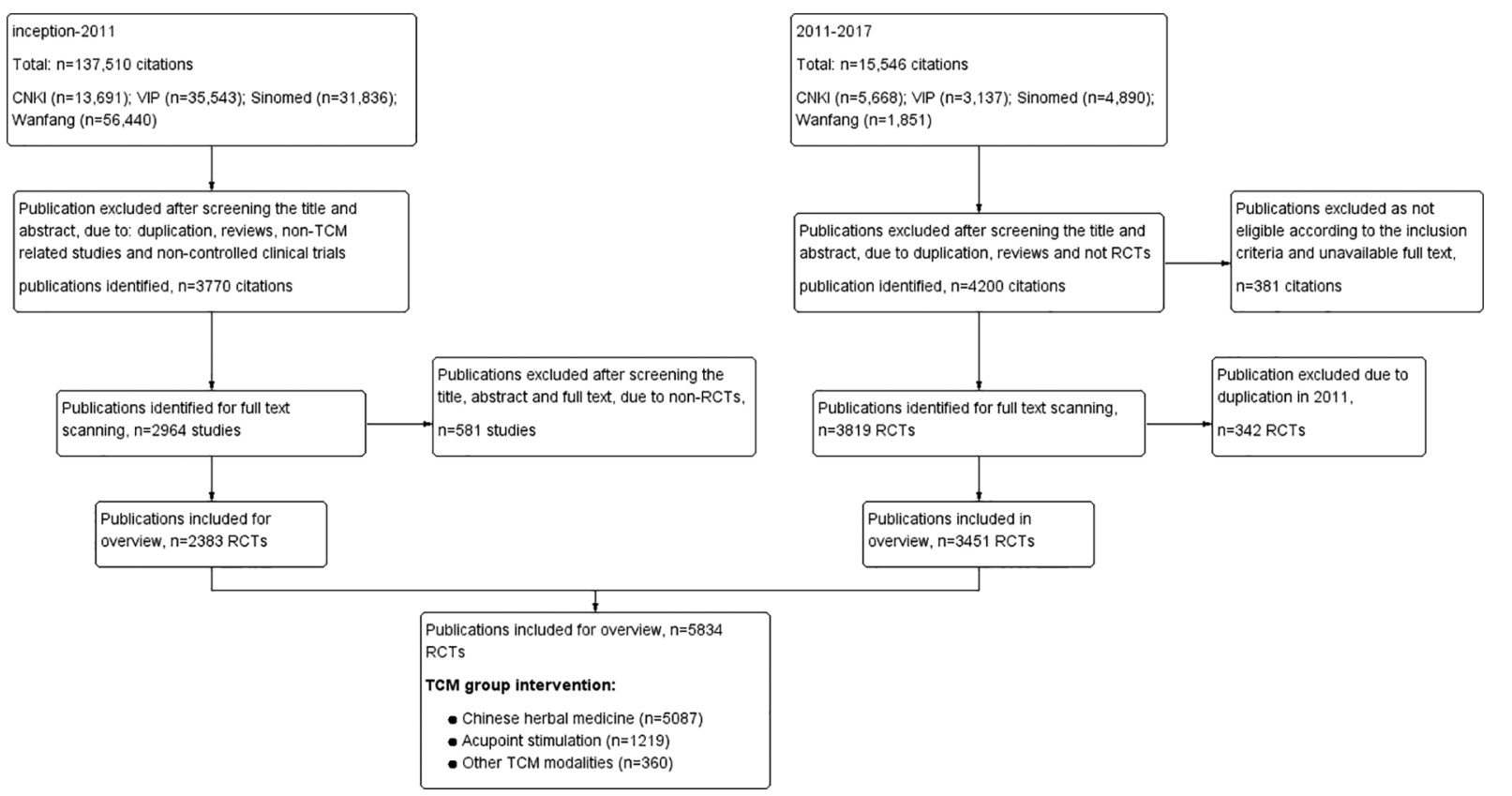

Figure I. Flow diagram of literature search and studies selected on TCM for cancer care published in Chinese (inception to 2017). Procedure of the literature search conducted between $201 \mathrm{I}$ and 2017, and study selection. The last block counted the study numbers of different traditional Chinese medicine (TCM) modalities in the group using TCM interventions.

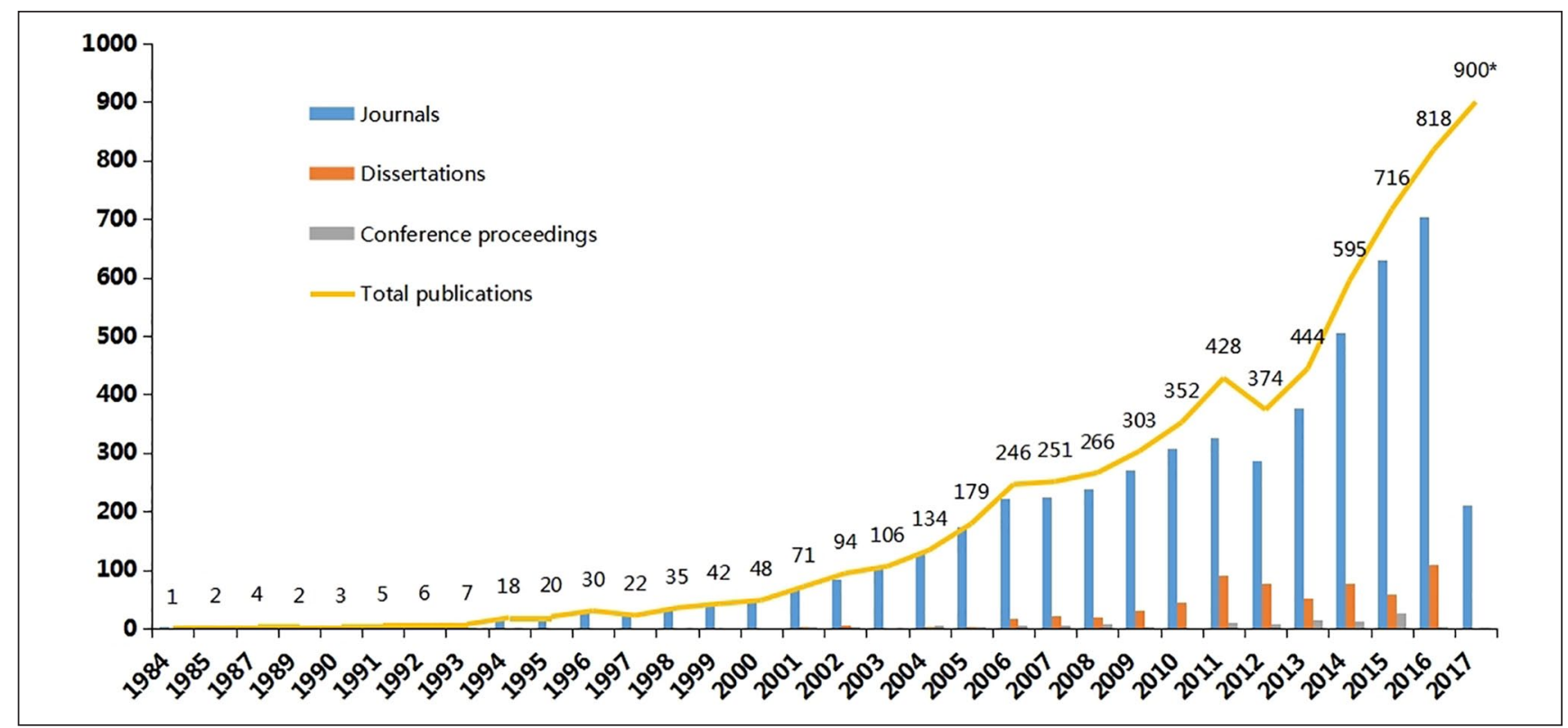

Figure 2. Publication category numbers of TCM RCTs for cancer published in Chinese from 1984 to 2017 (*estimated for 2017 ). Included studies were published or indexed as journal articles, dissertation (for masters and doctorates), and conference proceedings (only for full text). Searching was conducted in June 2017, so extrapolating the data to allow for a publication lag, it was estimated that there may be approximately up to 900 relevant RCTs for 2017.

Among the 5834 RCTs (477 157 participants), 157 studies $(2.69 \%)$ focused on precancerous conditions and the remainder focused on established cancer. A total of 2727
RCTs (46.74\%) reported the cancer stages. About 2315 studies $(39.68 \%)$ focused on the treatment of primary tumor directly, 553 studies $(9.48 \%)$ on cancer-related clinical 


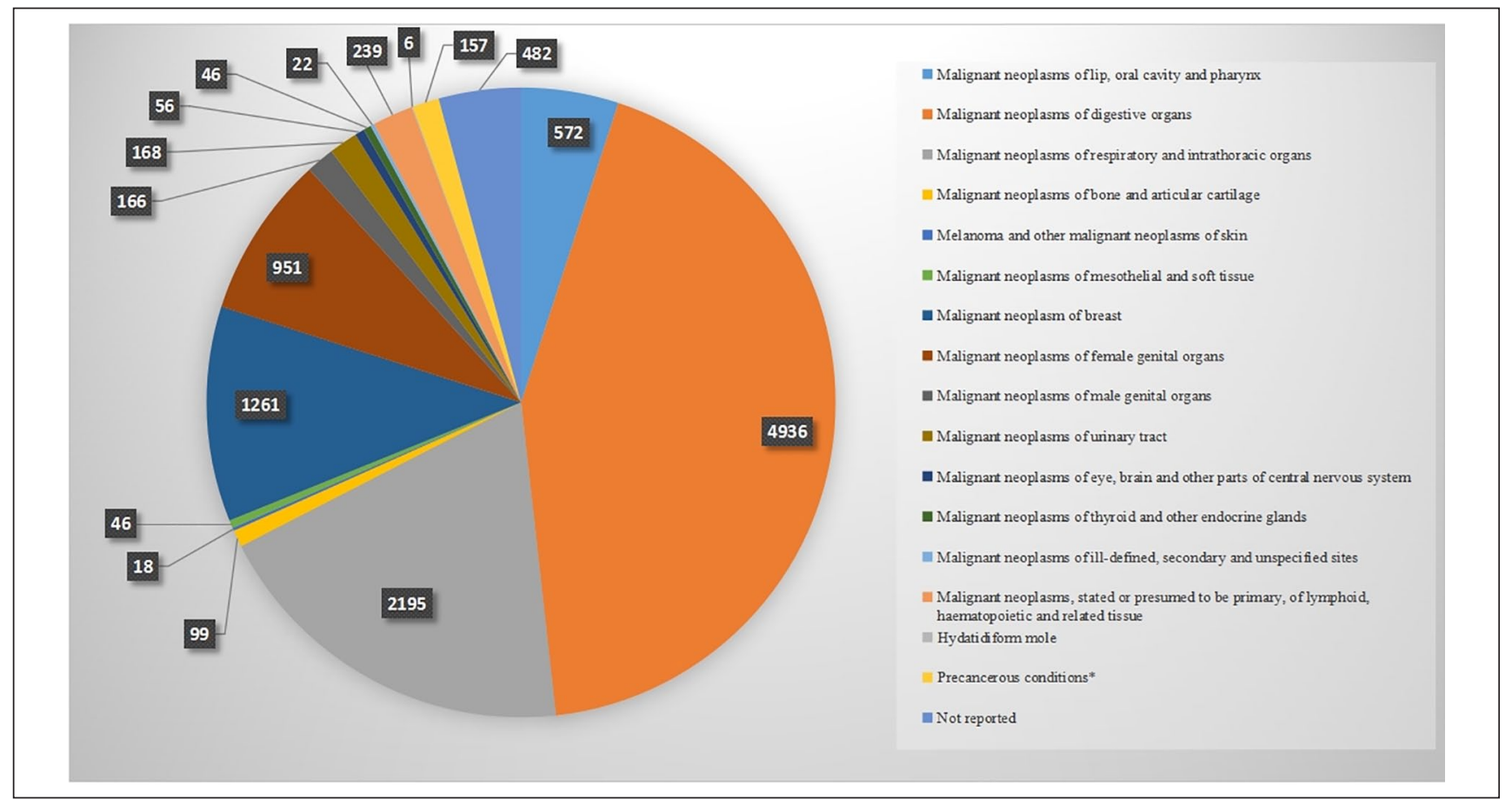

Figure 3. Numbers of TCM RCTs by cancer category published in Chinese. Cancer type reported in the included RCTs summarized into I 6 categories according to ICD-IO. Studies could be counted several times as I study may include more than I type cancer participant.

conditions like relapse and metastasis, 1847 studies $(31.66 \%)$ on the side effects of radio/chemotherapy, 559 studies $(9.58 \%)$ on post-surgery conditions like nursing care. Additionally, 363 studies (6.22\%) focused on the cancer related pain.

We grouped cancer type into 16 categories based on the International Classification of Disease 10 (ICD-10) (Supplemental Table 2, Figure 3). Malignant neoplasms of digestive organs (including stomach cancer, colorectal cancer, liver cancer etc.) were the most reported cancer types. There were also 482 studies which failed to report the type of cancer or could not be classified into an ICD-10 category, because patients were treated based on their symptoms. According to the numbers of cases and numbers of studies, the main 10 cancers treated with TCM were lung cancer, stomach cancer, breast cancer, colorectal cancer, liver cancer, esophagus cancer, nasopharyngeal carcinoma (including nasal carcinoma and nasal malignant granuloma), ovarian cancer, cervical cancer, lymphoma (Figure 4). Compared with the previous review, ${ }^{15}$ colorectal cancer has become the third most prevalent disease, and matches with the incidence of this cancer. ${ }^{2}$

\section{Interventions}

Of the total number of RCTs included (5834 RCTs), the majority of studies (5489 RCTs, 94.09\%) were designed with 2 arms, 294 RCTs (5.04\%) had 3 arms, and 51 RCTs
$(0.87 \%)$ had 4 or more arms. The treatment group was the intervention group for testing the effectiveness of TCM, and the control group acted as the standard treatment. In the treatment group, 4752 RCTs (81.45\%) used TCM combined with conventional treatment, whilst 1082 RCTs (18.55\%) used only TCM, mainly for symptoms and side effects rather than to treat cancer directly or halt disease progression. In the control group, 4969 RCTs $(85.17 \%)$ used only conventional treatment as their comparison, 550 RCTs $(9.43 \%)$ combined TCM with conventional treatment as the control, 302 RCTs (5.18\%) only with TCM, 13 RCTs $(0.22 \%)$ with no treatment as the control. Only 58 RCTs $(0.99 \%)$ reported using placebo as the control, mainly simulating the TCM acupoint sticking application and patent herbal medicine.

Most studies (2325 RCTs, 39.85\%) used TCM combined with chemotherapy, 1472 RCTs $(25.23 \%)$ used TCM combined with conventional medicine or other routine treatment for symptoms followed by dynamic and updated clinical guidelines, 502 RCTs $(8.60 \%)$ used TCM combined with radiotherapy, $227 \mathrm{RCTs}(3.89 \%)$ used TCM combined with interventional oncology therapy, 158 RCTs $(2.71 \%)$ used TCM combined with surgery in 1 group. In the other group (the control group), studies comparing controls with TCM or TCM modalities, radiotherapy was the main control treatment (3400 RCTs, 58.28\%), 2703 RCTs (46.33\%) applied conventional medicine or other routine treatment as the control, 219 RCTs (3.75\%) with interventional oncology therapy 


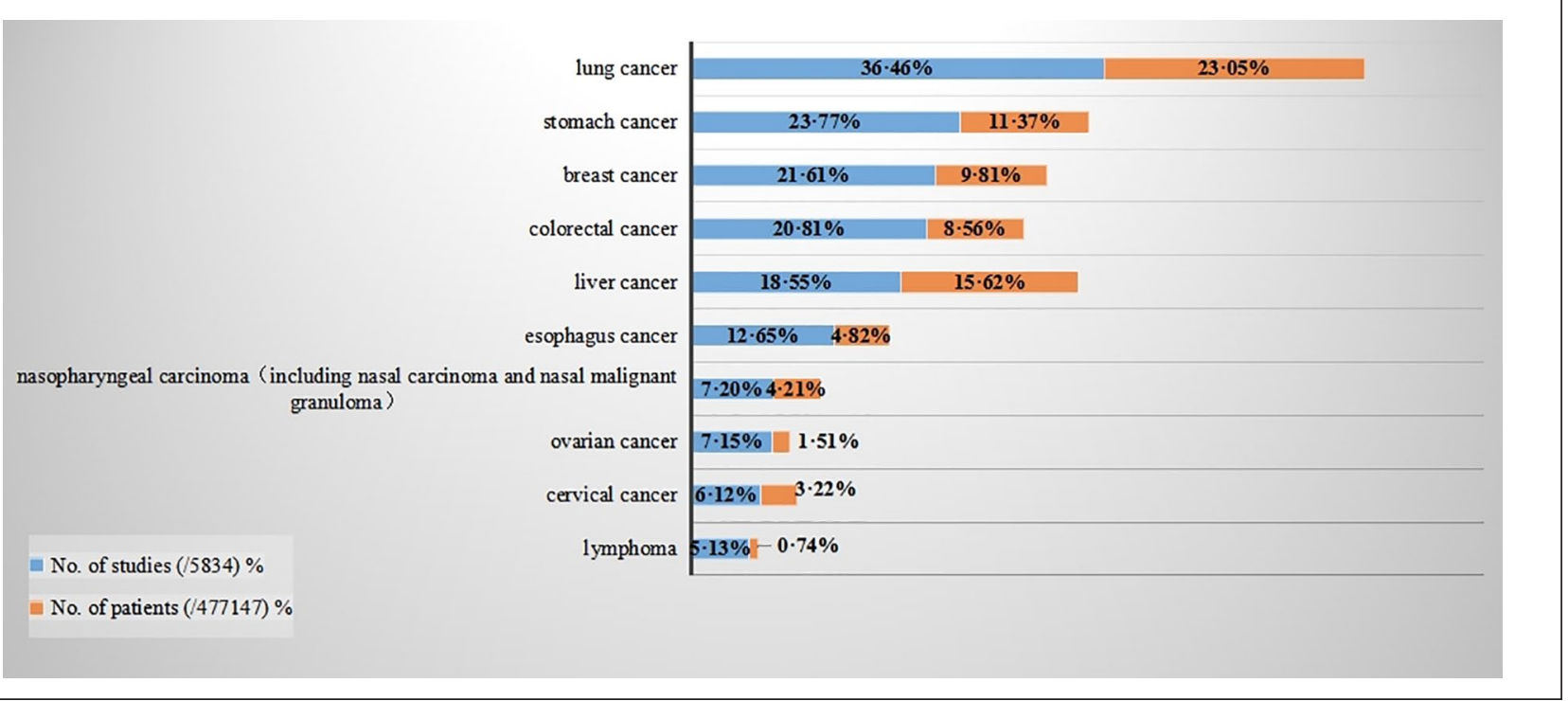

Figure 4. Top 10 cancer categories ranked by numbers of studies and patient numbers of TCM for cancer RCTs published in Chinese. Top 10 cancer types (according to ICD-I0) reported in the included RCTs were ranked by RCTs number. The top I0 numbers of studies of cancer types were also the top 10 numbers of patients; this is presented behind the numbers of studies.

as the control, $159 \mathrm{RCTs}(2.73 \%)$ had surgery as the control (for more details see Supplemental Table 3).

Across all trials, only 1757 RCTs (30.12\%) reported that the researchers diagnosed participants and prescribed the TCM treatment according to TCM syndrome differentiation. There were 7 TCM modalities, including Chinese herbal medicine, acupoint stimulation, Tuina (Chinese medical massage), dietary therapy, TCM psychological intervention, qigong/Tai chi, and TCM 5 element music therapy. Most trials used multiple TCM modalities based on TCM theory.

Herbal medicine. Among the 5834 RCTs, a total of 5087 RCTs $(87.20 \%)$ used Chinese herbal medicine in the treatment group, including proprietary herbal products (1368 RCTs, $23.45 \%$ ), hospital pharmacy prepared herbal medicine (391 RCTs, 6.70\%), and individualized practitioner prescription (2934 RCTs, 50.29\%). The methods of administration, listed in order of frequency, were oral medicine (3783 RCTs, 64.84\%), herbal injection (827 RCTs, 14.18\%), topical (638 RCTs, 10.94\%), and perfusion, foot bathing, nasal feeding, mouth rinsing, steaming and washing, intravaginal administration, or aerosol inhalation (for more details see Supplemental Table 2). When Chinese herbal medicine was used in the control group, proprietary herbal products (492 RCTs, $8.43 \%$ ) were the most frequently used Chinese herbal medicine category, and oral medication (437 RCTs, $7.49 \%$ ) was the most frequent method of administration (for more details see Supplemental Table 3).

Acupoint stimulation. A total of 1219 RCTs (20.89\%) used acupoint stimulation as their intervention. Among the 5834
RCTs, in order of frequency, the modalities of acupoint stimulation were needling (306 RCTs, 5.25\%), moxibustion (267 RCTs, 4.58\%), acupoint plaster application (215 RCTs, 3.69\%), acupoint injection (203 RCTs, 3.48\%), acupressure (152 RCTs, 2.61\%), ear acupuncture (122 RCTs, $2.09 \%$ ), and electro-acupuncture, point embedding therapy, laser or microwave stimulation, bee-venom therapy, cupping, or acupoint nerve stimulation. When acupoint stimulation was used in the control groups, needling (41 RCTs, $0.70 \%$ ) and acupoint injection (37 RCTs, $0.63 \%$ ) were most the frequently used treatments. Only 3 RCTs $(0.05 \%)$ used sham acupuncture as a comparison (more details see Supplemental Table 3).

Other TCM modalities. A total of 360 RCTs in the treatment group used other TCM modalities. Among the 5834 RCTs, in order of frequency, they were Tuina (Chinese medical massage) (133 RCTs, 2.28\%), dietary therapy (131 RCTs, $2.25 \%$ ), TCM theory based psychological intervention (44 RCTs, $0.75 \%$ ), qigong ( 31 RCTs, $0.53 \%$ ), and TCM 5 element music therapy ( $21 \mathrm{RCTs}, 0.36 \%$ ). When the control group used other TCM modalities, no RCTs used TCM 5 element music therapy, only 11 RCTs $(0.19 \%)$ used Tuina, 5 RCTs $(0.09 \%)$ used dietary therapy, 1 RCT $(0.02 \%)$ used TCM psychological intervention, 1 RCT $(0.02 \%)$ used qigong (more details see Supplemental Table 3).

\section{Outcome Measurement and Main Findings}

The most frequently reported outcome was clinical symptoms (3716 RCTs, $63.70 \%$ ), which was similar to the 2011 report. ${ }^{15}$ Among these clinical symptoms, 8 important 
clinical symptoms influencing the cancer patients' life, including pain (133 RCTs, 2.28\%), imaging indices (50 RCTs, $0.86 \%$ ), length of hospital stay (37 RCTs, $0.63 \%$ ), fever (35 RCTs, $0.60 \%$ ), urination (34 RCTs, $0.58 \%$ ), sleep (24 RCTs, $0.41 \%$ ), appetite (23 RCTs, $0.39 \%$ ), fatigue (17 RCTs, $0.29 \%$ ), economic indices (16 RCTs, $0.27 \%$ ), and TCM syndrome (9 RCTs, $0.15 \%$; details given in Table 1). Quality of life (2725 RCTs, 46.71\%) was a frequently measured outcome and the Karnofsky Score (1976 RCTs, $33.87 \%$ ) was the most widely used measurement scale. The other top 10 outcome measurements (Figure 5) were ranked as biomarker indices (2384 RCTs, $40.86 \%$ ), chemo/radiotherapy induced side effects (1977 RCTs, 33.89\%), tumor size (1541 RCTs, 26.41\%), safety (1299 RCTs, 22.27\%), survival (689 RCTs, 11.81\%), body weight (248 RCTs, $4.25 \%$ ), need for another operation (344 RCTs, 5.90\%), and the patients' values and mood (231 RCTs, 3.96\%). Patients' satisfaction (91 RCTs, $1.56 \%$ ) was a new outcome that emerged. The 1237 RCTs $(21.20 \%)$ reported multiple indices to provide a composite outcome to measure cancer patients' general condition. Based on these outcomes, for the majority of studies (4050 RCTs, 69.42\%) the authors concluded that compared with conventional treatment, TCM alone (647 RCTs, $11.09 \%$ ) or combined with conventional treatment (3403 RCTs, 58.33\%) showed a better effect in cancer care.

\section{Discussion}

This analysis updates a previous overview of studies in TCM and cancer published up to $2011 .{ }^{15}$ Given the rapid increase in publications, only RCTs were included as they provide the highest quality of evidence. Compared with the 2383 RCTs published between 1983 and 2011, the 3451 relevant RCTs published between 2011 and 2017 more than doubled, showing the popularity of this topic in Chinese research and practice.

\section{Summary of Findings}

Participants. All studies were conducted in China and enrolled Chinese people, and the cancer types most studied were broadly similar to the cancer prevalence in China according to the WHO in $2018^{16,17}$ (lung, colorectal, stomach, liver, and breast cancers). Compared with the previous overview, ${ }^{15}$ lung and stomach cancer still ranked as the top 2 cancer types most studied, breast cancer increased from fourth to third, and colorectal cancer increased from sixth to fourth. According to the incidence rates in the report of Cancer Statistics in China, ${ }^{17-20}$ breast and colorectal cancer are becoming bigger problems. The incidence of cervical cancer ranked seventh in China, and the number of the Chinese trials were also ranked at eighth. Although the nonavalent HPV (9vHPV) vaccines were produced to prevent the cervical cancer, ${ }^{20,21}$ there is still strong demand for prevention and cure. In addition, the number of liver cancer trials was ranked as fifth, but the number of the participants was still ranked as the second most studied. Liver cancer remains a common cancer in China.

Compared with RCTs published before 2011, ${ }^{15}$ the number of all kinds of cancer patients in RCTs being studied have more than doubled in the last 6 years. Among the 1144 studies reporting being funded, $76.12 \%$ (871 RCTs) were published in 2011 to 2017 , so these may be a result of Chinese government initiatives as they have focused on supporting TCM as an important research theme. Researchers have also had the chance to apply for more funding to conduct clinical research. ${ }^{22}$ Hence, the urgent need to update the current evidence to provide suggestions to support clinical practice and direct further research.

Interventions. Compared with RCTs published before $2011,{ }^{15}$ most RCTs (81.45\%) combined TCM and conventional medicine, with TCM therapies used to strengthen the effect of conventional treatment to reduce symptoms and side effects. ${ }^{23-30}$ Herbal medicines were still the main modality in cancer care, but all of the formulae still failed to report the ingredients, which makes it difficult to replicate and test the effectiveness and safety of interventions. The details of this research are important if it is to be replicated and used to inform patient care. Non-pharmacologic TCM therapies such as acupuncture stimulation, qigong, tuina (Chinese massage) have increased; it may be that non-pharmacologic TCM therapy may be perceived as safer and more acceptable in cancer care. Further studies should focus on non-pharmacologic TCM modalities to maintain cancer patients' quality of life.

Outcomes. Patients' mental health and satisfaction were included as several domains of outcomes in cancer RCTs. Increasingly there are studies which focus on patients' psychosocial state and attitudes. ${ }^{31,32}$ Relief of clinical symptoms is important, but improving cancer patients' psychological and mental condition to increase patients' quality of life should also be considered. Among the current RCTs, the Karnofsky Scale is most commonly used in the trials evaluating quality of life, while more complicated tools should be considered in the future research to evaluate patients' quality of life. Additionally, most of the RCTs showed that TCM had better effectiveness measured using a composite outcome, but there is still the need to objectively measure and identify their effect in the future well-designed studies.

Based on the mapping evidence, we found that Chinese patent medicine and Chinese medicine for oral administration were mostly used to treat the tumor itself, particularly in the case of lung and stomach cancer. Chinese medicine for external application was used mainly for cancer complications, acupuncture for complications after radiotherapy 
Table I. Outcome Measurements Reported in Cancers Treated by TCM in RCTs Published in Chinese.

\begin{tabular}{|c|c|c|c|}
\hline Outcome measurement & Description & Study No. & $\%(/ 5834)$ \\
\hline Clinical symptom & & 3716 & 63.70 \\
\hline \multirow[t]{21}{*}{ Quality of life } & & 2725 & 46.71 \\
\hline & Karnofsky Score & 1976 & 33.87 \\
\hline & EORTC-QLQ & 272 & 4.66 \\
\hline & QOL score without specific information & 169 & 2.90 \\
\hline & FACT & 78 & 1.34 \\
\hline & SF-36 & $4 I$ & 0.70 \\
\hline & ECOG Score & 35 & 0.60 \\
\hline & WHOQOL & 20 & 0.34 \\
\hline & $\begin{array}{l}\text { GQLI (Gastrointestinal Quality of Life } \\
\text { Index) }\end{array}$ & 8 & 0.14 \\
\hline & TCM quality of life scale & 7 & 0.12 \\
\hline & QLQ-CCC & 7 & 0.12 \\
\hline & EuroQLQ-BR23 & 4 & 0.07 \\
\hline & QLICP & 4 & 0.07 \\
\hline & DLQI & 3 & 0.05 \\
\hline & FLIC Scale & 3 & 0.05 \\
\hline & FLIC Scale & 2 & 0.03 \\
\hline & Barthel Index & 2 & 0.03 \\
\hline & Integrative medicine quality of life scale & 2 & 0.03 \\
\hline & QOL Scale-FAMILY & I & 0.02 \\
\hline & Other & 19 & 0.33 \\
\hline & Not reported & 70 & 1.20 \\
\hline Biomarker indices & & 2384 & 40.86 \\
\hline Chemo/radiotherapy induced side effects & & 1977 & 33.89 \\
\hline \multirow[t]{6}{*}{ Tumor } & & 1877 & 32.17 \\
\hline & Tumor size & |54| & 26.41 \\
\hline & Tumor relapse & 172 & 2.95 \\
\hline & Tumor metastasis & 157 & 2.69 \\
\hline & Tumor numbers & 4 & 0.07 \\
\hline & $\begin{array}{l}\text { Cancerization rate in precancerous } \\
\text { conditions }\end{array}$ & 3 & 0.05 \\
\hline Safety & & 1299 & 22.27 \\
\hline Survival & & 689 & 11.81 \\
\hline Body weight & & 248 & 4.25 \\
\hline Body measurement & & 29 & 0.50 \\
\hline \multirow[t]{5}{*}{ Operation } & & 344 & 5.90 \\
\hline & Preoperative complications & 2 & 0.03 \\
\hline & Intraoperative care & 2 & 0.03 \\
\hline & Postoperative side effects & 235 & 4.03 \\
\hline & Postoperative care & 105 & 1.80 \\
\hline \multirow[t]{4}{*}{ Prognosis } & & 144 & 2.47 \\
\hline & Prognosis medicine & 74 & 1.27 \\
\hline & Prognosis treatment & 68 & 1.17 \\
\hline & Prognosis factors & 2 & 0.03 \\
\hline \multirow{6}{*}{ Patients' mental health and satisfaction } & & 231 & 3.96 \\
\hline & Depression indexes & 74 & 1.27 \\
\hline & Anxiety mood & 50 & 0.86 \\
\hline & Psychological states & 7 & 0.12 \\
\hline & Patients' satisfaction & 91 & 1.56 \\
\hline & Patients' compliance & 9 & 0.15 \\
\hline
\end{tabular}


Table I. (continued)

\begin{tabular}{lrr}
\hline Outcome measurement & Description & Study No. \\
\hline Other important clinical symptom (not & \\
$\quad$ included in clinical symptom part) & $133834)$ \\
Pain & 50 \\
Imaging indexes & 37 \\
Hospital stay length & 0.28 \\
Fever & 35 \\
Urination & 34 \\
Sleep & 0.63 \\
Appetite & 0.60 \\
Fatigue & 0.58 \\
Economic indices & 0.41 \\
TCM syndrome & 24 \\
\hline
\end{tabular}

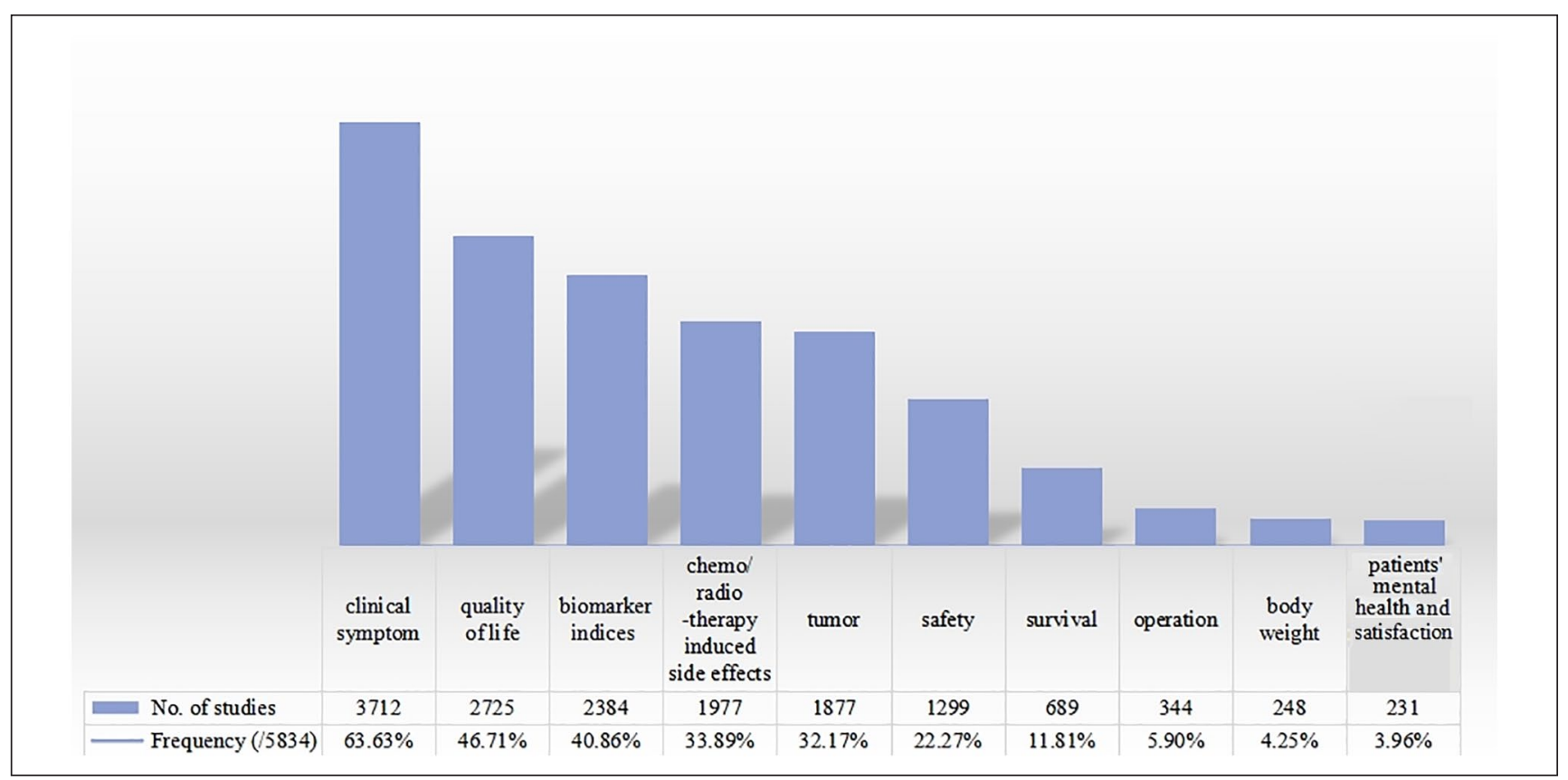

Figure 5. Top 10 outcome measurements reported in cancer treated by TCM in RCTs published in Chinese. Outcome measurements reported in the included RCTs were calculated by the study number (more details in Table I). They could be counted several times as one study may have included more than one outcome.

and chemotherapy, and both tuina (Chinese massage) and qigong mostly in the postoperative recovery phase. In the future, we can test different TCM modalities for different conditions to compare their advantages.

\section{Comparison with Previous Studies}

There has been no comprehensive descriptions of Chinese research on TCM for cancer care other than our previous studies in this area. ${ }^{15,33-35}$ We have combined the information from our earlier overview with updated information on more recent RCTs and summarized the characteristics and trends in this research area. It is our hope that this can provide information on both the strengths and weaknesses of this research, and identify gaps in the research agenda for these commonly used interventions.

This current paper only included RCTs, which were considered as the high-quality research, and has been extended to include RCTs published in 2011 to 2017. This update has included an additional 3451 RCTs, which has more than doubled compared with the previous study.

\section{Strengths and Limitations}

A large number of RCTs of TCM for cancer care have been published in Chinese, mostly without an English abstract, 
and few are indexed in MEDLINE or other English databases. It is problematic for people outside of China to access this evidence. Since our previous study in 2011 attracted wide attention, this update provides important information for people who cannot access and read Chinese literature, and could be used to identify potentially effective areas for research into TCM and cancer.

There are also some limitations to our work, which also provide the basis for our next steps. As the aim of this paper was to describe the general scope of TCM assessed in cancer RCTs rather than provide a detailed evaluation, we unfortunately did not set a high quality or strict criteria. Regarding searching, due to the large number of updated RCTs, we only searched the publications in Chinese, and English databases were not searched. High-quality RCTs designed and conducted by Chinese people and TCM practitioners may be have been published in the English language journals, and these may have been missed. Another review focusing on these studies will be conducted, and therefore improve our bibliometric analysis by using a dynamic database in order to update the data yearly. In our study, we included all kinds of TCM used in RCTs of cancer care. These RCTs present an overall distribution of current TCM RCTs published in Chinese, though the inclusion criteria did not include an assessment of study quality. Compared with the previous study which included both randomized and non-randomized controlled trials, we only focused on randomized controlled trials given the increased number of published studies and the fact that these studies were likely to provide a higher level of evidence given their study design. It is necessary to provide the robust evidence to inform clinical practice, so we suggest future studies should focus more specifically on PICO (Participant, intervention, comparison, and outcome) questions and studies with higher quality as these will provide stronger data on the evidence of effectiveness and safety. For quality assessment of included trials, we have just reported the number of included trials methodology without further assessment; future work will evaluate the risk of bias to discover the temporal trend of quality of TCM trials. For key elements of trials (participants, intervention, comparison, outcomes), this overview is not the whole picture for RCTs of TCM for cancer care in the Chinese literature, and cannot show the detailed relationship among participants, interventions, comparisons, and outcomes. Our next steps will involve another project to map the structure of RCTs according to participants, interventions, comparisons and outcomes, and to explore the possible and specific trends in research and the knowledge gaps, especially advantages of TCM for different cancer types, cancer stages, and patients' condition. For details on TCM modalities and evidence-based evaluation, we failed to analyze the origin of herbs, quality control, side effects of the TCM treatments, but TCM has its own principles and rules to prescribe the formula of herbal medicine and acupoints selection. So regarding these limitations, we will also summarize all reported TCM formulae and methods of implementation in RCTs to provide data on repeatability of trials based on TCM theory. Several systematic reviews based on the current literature will be conducted to evaluate the effectiveness and safety of TCM, such as TCM patents, qigong, acupoint stimulation, which will be more feasible and applied to guide future RCT and clinical practice.

\section{Implications for Research}

Based on our studies, the knowledge gaps can be identified to indicate the future RCTs required and their relevance to clinical practice. From the aspect of participants, TCM in these trials showed potential advantages on cancer itself and side effects of radio/chemotherapy. Regarding interventions, common TCM interventions like Chinese patent medicine and acupuncture appeared to be more accessible, applicable and repeatable for cancer care. The effectiveness of most TCM modalities for cancer care needs to be confirmed. In terms of comparing integrative therapy, TCM plus conventional treatment, potential advantages were indicated for improving clinical symptoms. Further evaluation of the beneficial effects and safety of a specific TCM modality is needed, especially herbal medicine according to specific conditions or stage of cancer. This will require conducting high quality RCTs and systematic reviews. Core outcomes especially survival, clinical symptoms, and quality of life should be considered as priority to reflect the effectiveness of TCM for cancer. Cancer patients' expectation, mood, and value when they are taking TCM should be explored.

Therefore, further TCM studies need to be improved in terms of design and reporting as the first basic step. There is also a need for clearer diagnostic and inclusion criteria to clarify the included populations and understand how to incorporate TCM syndromes in the choice and design of TCM modality. The common TCM interventions in cancer care, measured by core outcomes especially the specific symptoms of side-effects, and patients' mood to confirm the effectiveness of TCM will be researched. For some composite outcomes used in TCM scales, ICD-11 will help to diagnose TCM syndromes and specific outcomes used to validate the specificity of TCM for cancer. More attention on patients' expectation, mood, and value to promote share decision making in clinical practice should be considered. The promising effects of TCM interventions need to be confirmed through rigorous research involving both the TCM and the conventional research communities, and therapies that prove effective should be developed and disseminated worldwide. 


\section{Conclusion}

Substantial data from 5834 RCTs published in Chinese showed that different types and stages of cancer patients were treated with many different TCM modalities either as mono-therapy for palliative care or in combination with conventional medicine for cancer prevention and treatment. These were measured by various outcomes. We need further comprehensive evaluation of the beneficial effects and safety of these TCM modalities, and we should focus on quality of life, and explore the reasons why cancer patients take TCM.

\section{Acknowledgments}

Much appreciation goes to all authors involved in the previous review, Guo-yan Yang, Xin-xue Li, Yan Zhang, Jing-li Yang, Jiu Chang, Xiao-xuan Sun, Xiao-yun Zhou, Yu Guo, Yue Yu, Alan Bensoussan for their whole-hearted input and contribution.

\section{Author Contributions}

$\mathrm{XL}$ and JPL conceived the study and designed the study. CLL analyzed the data and drafted manuscript. CLL, YYY, and CZ conducted the searching in 4 databases in 2017. CLL, HMZ selected the RCTs from the previous review. ${ }^{14} \mathrm{XL}$ and JPL finalized the data extraction form. XL and CLL monitored the data extraction procedure. CLL, CZ, HMZ, YYY, JMD, and YJG downloaded and examined the full text from the Internet. HMZ, CZ, YYY, RLF, CJL, FYD, JCL, ZMC, QYM, JPZ, YFH, SYH, JYQ, YXLi, YW, YQY, JMD, YXLu, YMC, and YJG performed the study including screened papers and extracted the data. NR, LSW, JPL, and XL revised and commented on the drafted manuscript. All authors approved the final manuscript.

\section{Declaration of Conflicting Interests}

The author(s) declared no potential conflicts of interest with respect to the research, authorship, and/or publication of this article.

\section{Funding}

The author(s) disclosed receipt of the following financial support for the research, authorship, and/or publication of this article: This work was supported by the Key project of the National Natural Science Foundation of China (No. 81830115). Prof. Nicola Robinson (visiting professor of Beijing University of Chinese Medicine) is funded by International development and capacity enhancement of evidence-based Chinese medicine Project, Ministry of Science and Technology of the People's Republic of China (G20200001187).

\section{ORCID iD}

Chun-Li Lu (iD https://orcid.org/0000-0003-2939-1327

\section{Supplemental Material}

Supplemental material for this article is available online.

\section{Reference}

1. Ferlay J, Ervik M, Lam F, et al. Global cancer observatory: cancer today. Lyon, France: International Agency for Research on Cancer. 2018. Accessed March 10, 2019. https:// gco.iarc.fr/today.

2. Bray F, Ferlay J, Soerjomataram I, Siegel RL, Torre LA, Jemal A. Global cancer statistics 2018: GLOBOCAN estimates of incidence and mortality worldwide for 36 cancers in 185 countries. CA Cancer J Clin. 2018;68:394-424.

3. World Health Assembly, World Health Organization. Cancer prevention and control in the context of an integrated approach. 2017. Accessed March 10, 2019. http://www.who. int/iris/handle/10665/275676.

4. European society for medical oncology. A landmark for cancer on the global health agenda and one of the most important documents on cancer of our time. 2017. Accessed March 10, 2019. https://www.esmo.org/Policy/Policy-News/2017WHO-Cancer-Resolution-Cancer-prevention-and-control-inthe-context-of-an-integrated-approach.

5. Miller KD, Siegel RL, Lin CC, et al. Cancer treatment and survivorship statistics, 2016. CA Cancer J Clin. 2016;66: 271-289.

6. International Activities. 2016. Accessed April 30, 2021. https://cam.cancer.gov/default.htm.

7. Are You Considering a Complementary Health Approach? 2016. Accessed March 10, 2019 https://nccih.nih.gov/health/ decisions/consideringcam.htm.

8. Cheung F. TCM: made in China. Nature. 2011;480:S82-S83.

9. Greenlee H, Balneaves LG, Carlson LE, et al. Clinical practice guidelines on the use of integrative therapies as supportive care in patients treated for breast cancer. J Natl Cancer Inst Monogr. 2014;50:346-358.

10. Paul E G, Kathrin S W, Brittany L, et al. Challenges to effective cancer control in China, India, and Russia. Lancet Oncol. 2014;15(5):489-538.

11. Lam WC, Zhong L, Liu Y, et al. Hong Kong Chinese medicine clinical practice guideline for cancer palliative care: pain, constipation, and insomnia. eCAM. 2019;2019:1038206.

12. Chung V. C, Wu X, Lu P, et al. Chinese herbal medicine for symptom management in cancer palliative care: systematic review and meta-analysis. Medicine. 2016;95(7):e2793.

13. Fu B, Wang N, Tan H. Y, et al. Multi-component herbal products in the prevention and treatment of chemotherapy-associated toxicity and side effects: a review on experimental and clinical evidences. Front Pharmacol. 2018;9:1394.

14. Noh H, Yoon S. W, Park B. A systematic review of herbal medicine for chemotherapy induced peripheral neuropathy. eCAM. 2018;2018:6194184.

15. Li X, Yang G, Li X X, et al. Traditional Chinese medicine in cancer care: a review of controlled clinical studies published in Chinese. PloS One. 2013;8(4): e60338.

16. Ferlay J, Colombet M, Soerjomataram I, et al. Estimating the global cancer incidence and mortality in 2018: GLOBOCAN sources and methods. Int J Cancer. 2019;144(8):1941-1953.

17. Feng RM, Zong YN, Cao SM, Xu RH. Current cancer situation in China: good or bad news from the 2018 Global Cancer Statistics? Cancer Commun (Lond). 2019;39(1):22. 
18. Sun KX, Zhang RS, Zhang SW, et al. Report of cancer incidence and mortality in different areas of China: 2015. China Cancer. 2019;28(01):1-11.

19. Chen W, Sun K, Zheng R, et al. Cancer incidence and mortality in China, 2014. Chin J Cancer Res. 2018;30(1):1-12.

20. Chen WQ, Sun KX, Zhang RS, et al. Report of cancer incidence and mortality in different areas of China: 2014. China Cancer. 2018;27(1):1-14.

21. Petry KU, Bollaerts K, Bonanni P, et al. Estimation of the individual residual risk of cervical cancer after vaccination with the nonavalent HPV vaccine. Hum Vaccin Immunother. 2018;14(7):1800-1806.

22. Xinhua News Agency. The state administration of traditional Chinese medicine (TCM) released the top 10 TCM news in 2011. 2012. Accessed March 10, 2019. http://www.gov.cn/ jrzg/2012-01/16/content_2045782.htm

23. Yeung KS, Gubili J, Mao JJ. Herb-drug interactions in cancer care. Oncology (Williston Park). 2018;32(10):516-520.

24. Xiao Z, Wang C, Zhou M, et al. Clinical efficacy and safety of Aidi injection plus paclitaxel-based chemotherapy for advanced non-small cell lung cancer: a meta-analysis of 31 randomized controlled trials following the PRISMA guidelines. J Ethnopharmacol. 2019;228:110-122.

25. Chen W, Wang Y, Liang Q, et al. Efficacy and safety of Aidi injection combined with transcatheter arterial chemoembolization on primary hepatic carcinoma: a systematic review and meta-analysis. Evid Based Complement Alternat Med. 2018;2018:6376429.

26. Wu J, Zhang D, Ni M, et al. Effectiveness of Huachansu injection combined with chemotherapy for treatment of gastric cancer in China: a systematic review and Meta-analysis. $J$ Tradit Chin Med. 2020;40(5):749-757.
27. Chen H, Yao X, Li T, et al. Compound Kushen injection combined with platinum-based chemotherapy for stage III/IV non-small cell lung cancer: a meta-analysis of 37 RCTs following the PRISMA guidelines. J Cancer. 2020;11(7):18831898.

28. Kennedy DA, Seely D. Clinically based evidence of drugherb interactions: a systematic review. Expert Opin Drug Saf. 2010;9(1):79-124.

29. Fasinu PS, Rapp GK. Herbal interaction with chemotherapeutic drugs-a focus on clinically significant findings. Front Oncol. 2019;9:1356.

30. Ben-Arye E, Lavie O, Samuels N, et al. Safety of herbal medicine use during chemotherapy in patients with ovarian cancer: a "bedside-to-bench" approach. Med Oncol. 2017;34(4):54.

31. Philipp R, Mehnert A, Müller V, Reck M, Vehling S. Perceived relatedness, death acceptance, and demoralization in patients with cancer. Support Care Cancer. 2019;28(6):2693-2700.

32. Robinson S, Kissane DW, Brooker J, Burney S. A systematic review of the demoralization syndrome in individuals with progressive disease and cancer: a decade of research. J Pain Symptom Manage. 2015;49(3):595-610.

33. Jin X, Ruiz Beguerie J, Sze DMY, Chan GCF. Ganoderma lucidum (Reishi mushroom) for cancer treatment. Cochrane Database Syst Rev. 2016;4(4):CD007731.

34. Zhang M, Liu X, Li J, He L, Tripathy D. Chinese medicinal herbs to treat the side-effects of chemotherapy in breast cancer patients. Cochrane Database Syst Rev. 2007;2:CD004921.

35. Chen X, Deng L, Jiang X, Wu T. Chinese herbal medicine for oesophageal cancer. Cochrane Database Syst Rev. 2016;1:CD004520. 